\title{
Biosynthesis, characterization, and antibacterial activity of silver nanoparticles from Aspergillus awamori
}

\author{
Vishwanatha $\mathrm{T}^{* 1}$, Keshavamurthy $\mathrm{M}^{2}$, Mallappa $\mathrm{M}^{3}$, Murugendrappa $\mathrm{M} \mathrm{V}^{4}$, Nadaf Y F ${ }^{5}$, Siddalingeshwara K G ${ }^{6}$, Dhulappa A \\ ${ }^{1}$ P.G Department of Microbiology, Maharani’s Science College for Women, Bengaluru - 560 001, Karnataka, India. ${ }^{2}$ Department of Post Graduate Studies and \\ Research in Microbiology, Gulbarga University, Kalaburagi - 585 106, Karnataka, India. ${ }^{3}$ Department of Chemistry, Maharani's Science College for Women, \\ Bengaluru - 560 001, Karnataka, India. ${ }^{4}$ Department of Physics, B.M.S College of Engineering, Bengaluru - 560 019, Karnataka, India. ${ }^{5}$ P.G. Department \\ of Physics and Research center, Maharani's Science College for Women, Bengaluru - 560 001, Karnataka, India. ${ }^{6}$ Scientific and Industrial Research Center, \\ Bengaluru - 560 022, Karnataka, India.
}

\section{ARTICLE INFO}

Article history:

Received on: January 29, 2018

Accepted on: March 14, 2018

Available online: August 01, 2018

\section{Key words:}

Silver nanoparticles,

Aspergillus awamori,

Bioreduction,

Antibacterial activity,

Fourier transform-infrared

spectroscopy.

\section{ABSTRACT}

Microbial nanoparticles (NPs) have become the subject of immense research interest in the recent past due to their wide range of applications as effective antimicrobial agents, drug delivery systems, gene delivery, diagnostic agents in imaging diseases, and consumer products among others. The present study emphasizes on the synthesis of metallic silver NPs (SNPs) from cell-free supernatant of Aspergillus awamori strain KGSR12. The phase purity, composition, size, and shape of the as-synthesized NPs were characterized using various analytic spectroscopic techniques including X-ray diffraction, scanning electron microscope (SEM), ultraviolet-visible, and Fourier transform-infrared spectroscopy. Based on the SEM analysis, the particles are uniformly distributed, and size is estimated to be $40-50 \mathrm{~nm}$. Antibacterial activity of NPs against significant human pathogens was conferred with well diffusion assay, and it reveals the strains of Pseudomonas aeruginosa, Klebsiella pneumoniae, and Bacillus cereus are susceptible to synthesized SNPs that confirm the antibacterial activity of SNPs. Thus, the study concludes with the biogenic and eco-friendly route for synthesizing SNPs with antibacterial activity against clinically important pathogens and attributes growing interest on fungi as an emerging source for the synthesis of NPs.

\section{INTRODUCTION}

Nanotechnology is a burgeoning area of interest in the recent years due to the production and development of materials at the nanoscale. The development of nanoparticles (NPs) has been an emerging highlight of nanotechnology [1]. Materials developed at nanoscale level are used in various fields such as optical sensors, electronic devices, biomarkers, catalysis, antimicrobial agents, and drug delivery devices [2]. Nanotechnology has made rapid progress in the recent era, which is highly significant in the competitive scenario. NPs exhibit intriguing physicochemical and biological properties due to large surface to volume ratio, size, shape, and tunable band gap. Nanotechnology has opened new avenues by intersecting with interdisciplinary field of science for innumerable applications [3]. Hence, nanomaterials research has gained significant interest in the recent years.

Silver NPs (SNPs) have become the subject of immense research interest in the recent years due to their wide range of applications as antimicrobial agents, water purification systems, medical devices,

*Corresponding Author:

Vishwanatha T, P.G Department of Microbiology, Maharani's Science College for Women, Bengaluru - 560 001, Karnataka, India.

E-mail:lvphaget@rediffmail.com cosmetics, and other consumer products [4]. Due to their high surface to volume ratio, they also possess specific physiochemical properties compared to bulk material. One of the crucial aspects of nanotechnology relies on the NPs synthesis process. It can be synthesized through physical, chemical, and biological means. High energy consumption and use of toxic chemicals limit the use of physical and chemical synthesis methods, respectively. Hence, an alternative approach based on biogenic principles can be employed for safe, cost-effective, and eco-friendly process to synthesize NPs using biological entities and its metabolites [5].

Previous work by many researchers reported that SNPs bear antimicrobial property against pathogenic microorganisms. SNPs exhibit a different mode of action ranging from cell wall damage to interacting with DNA molecule and vital enzymes responsible for cell metabolic activity and survival. In recent years, SNPs have become the focus of intensive research due to their wide range of applications in areas such as catalysis, optics, biomarkers, and antimicrobials [6]. Depending on their size shape and distribution they exhibit various properties. Different approaches have been used for the synthesis of NPs [7]. In recent past, a great deal of intensive effort has been put into the synthesis of NPs using plants and microorganisms. Fungi, bacteria, yeasts, and several other microbes have shown to have the 
ability to reduce metal ions into metallic NPs [8]. Among microbes, fungi are versatile organism's exhibit immense tolerance to metal salts and also have good ability to secrete extracellular enzymes which act as a reducing and capping agents. Fungal mediated synthesis of SNPs has been done by a number of researchers so far. Fusarium oxysporum [7], Aspergillus fumigates [9], Fusarium semitectum [10], Aspergillus niger [11], Cladosporium cladosporioides [12], Penicillium brevicompactum [13], and Aspergillus clavatus [14] have been previously described. An extensive intracellular and extracellular study has been conducted on the biosynthesis of SNPs by Aspergillus species.

In the present study, SNPs were synthesized using Aspergillus awamori for the reduction of $\mathrm{Ag}^{+}$ions to $\mathrm{Ag}^{\circ} \mathrm{NPs}$ within $24 \mathrm{~h}$ of reaction time at ambient temperature. Further, biosynthesized SNPs are found to be highly effective against Pseudomonas aeruginosa, Klebsiella pneumoniae, and Bacillus cereus. To the best of our knowledge, this is the first report to synthesize the NPs and its effect against pathogenic bacteria from the fungi A. awamori.

\section{EXPERIMENTAL SECTION}

\subsection{Biosynthesis and Characterization of Microbial NPs (MNPs)}

All the chemicals employed in the present investigation were procured from HiMedia and Sigma-Aldrich, Bengaluru, India. Silver nitrate was obtained from Sigma-Aldrich. Nutrient agar and Mueller Hinton agar (MHA) were obtained from HiMedia. Pure culture of A. awamori (KGSR12) was collected from the Scientific Industrial Research Laboratory, Bengaluru, India [15]. The fungus obtained was routinely maintained in the laboratory on Potato Dextrose Agar and incubated at $28^{\circ} \mathrm{C}$ with regular subculturing on fresh media. The fungus A. awamori (KGSR12) was grown in $250 \mathrm{~mL}$ Erlenmeyer flask containing $100 \mathrm{~mL}$ potato dextrose broth (potato extract - $20 \%$, dextrose $-2 \%$, peptone $-1 \%$, and $\mathrm{pH}$ ) and incubated at $28^{\circ} \mathrm{C}$ for $96 \mathrm{~h}$. After incubation, the biomass was filtered through Whatman filter paper No. 1. Different volumes of fungal supernatant and $1 \mathrm{mM}$ $\mathrm{AgNO}_{3}(\mathrm{w} / \mathrm{v})$ was mixed, and the reaction mixture was incubated in dark condition at room temperature for bioreduction. The bioreduction of metal ions in the reaction mixture was screened by sampling the aliquots at different time intervals.

After addition of fungal filtrate to $\mathrm{AgNO}_{3}$, tubes containing the reaction mixture were kept for visual observation for color change. Metal ion reduction in the reaction mixture was monitored by visual observation and further confirmed by ultraviolet (UV)-visible spectroscopy (UV1800, Shimadzu, Japan) analysis as another preliminary test for the presence of SNPs in the wavelength range of 200-600 nm. The surface plasmon resonance peak was assessed for size and dispersity of the synthesized colloidal SNPs. Double distilled water was used as a blank. The colloidal suspension of SNPs was lyophilized and dried into fine powder and used for Fourier transform-infrared (FT-IR) and X-ray diffraction (XRD). A FT-IR spectrum of the as-synthesized nanoparticle was monitored over the range of $4000-400 / \mathrm{cm}$ using FT-IR spectroscopy (Agilent carry-630) to examine the presence of functional moiety. The crystal structure, phase purity, and crystallinity size of the as-synthesized NPs were performed by X-ray diffractometer PANalytical X'pert PRO X-ray diffractometer operated at $30 \mathrm{kV}$, and a current of $15 \mathrm{~mA}$ with $\mathrm{CuK} \alpha$ radiation $\left(\lambda=1.5406 \mathrm{~A}^{\circ}\right)$ and the $2 \theta$ scanning range was of $10-80^{\circ}$ at $5^{\circ} \mathrm{min}$. The morphology and their elemental composition of the as-synthesized NPs were analyzed using scanning electron microscope (SEM) associated with energy dispersive X-ray (EDX) (TESCAN Vega 3 LMU) operated at 5-30 kV of resolution $3 \mathrm{~nm}$ at $12.260 \mathrm{keV}$.

\subsection{Antibacterial Activity of MNP}

The SNPs synthesized from A. awamori were tested for antibacterial activity. The antibacterial activity determined using the well diffusion method against pathogenic organisms such as $P$. aeruginosa, $K$. pneumoniae, and B. cereus. All the bacterial pathogens used in the present study were isolated from clinical samples. The bacterial cultures were inoculated into Luria Bertani broth and incubated at $37^{\circ} \mathrm{C}$ to get moderate turbidity obtained. Thus obtained was matched with 0.5 McFarland's standard. The cultures swabbed uniformly on the MHA plates using a sterile cotton swab. Sterile Cork borer $(6 \mathrm{~mm}$ diameter) was used to make wells on solidified media. $50 \mu \mathrm{L}$ of SNPs were pipetted into wells labeled as SNP. Two control wells (C1 - fungal filtrate and $\mathrm{C} 2$ - deionized water) were made, and all the plates were incubated at $37^{\circ} \mathrm{C}$ for $24 \mathrm{~h}$. After incubation antimicrobial property of SNPs was determined by measuring the zone of inhibition around the wells in diameter (millimeter). Chloramphenicol, a standard antibiotic of concentration $200 \mu \mathrm{g} / \mathrm{ml}$, was used as the positive control (PC).

\section{RESULTS AND DISCUSSION}

In the present study, to determine the effect of different volumes of cells free extract (filtrate) of $A$. awamori, we treated fungal supernatant with different volume of $1 \mathrm{mM}$ silver nitrate solution at room temperature. The synthesis of metallic SNPs was initially confirmed with a change in color of the solution from pale yellow to orange color. Figure 1 shows color change in the experimental tube (Test 3-6 mL of silver nitrate solution plus $4 \mathrm{~mL}$ of fungal filtrate) from pale yellow to orange color in with $1 \mathrm{mM} \mathrm{AgNO}_{3}$ concentration, which is considered as optimized volume based on absorption peak in the UV-visible spectra. The color change remained stable for several days at $4^{\circ} \mathrm{C}$. Although the color in the reaction mixture intensifies with regard to time, temperature, and concentration of silver nitrate, no color change was observed in the tubes maintained as controls. Previous works on NPs reported that the change in color of NPs in aqueous solution is due to vibrations of surface plasmon exhibited by NPs $[1,16]$. Further confirmation of metallic NPs was carried out by UV-visible spectroscopic analysis [Figure 2] with absorbance peak emerging between 300 and $600 \mathrm{~nm}$ wherein different spectral lines indicate a synthesis of metallic NPs at different volumes. Thus, our result provides a promising spectroscopic signature of nanoparticle formation. The synthesis was evidently expressed by the bioreduction of silver ions into corresponding NPs which may be due to the presence of the nicotinamide adenine dinucleotide hydrogen-dependent enzyme, nitrate reductase that probably acts as an electron carrier in the reduction process [7]. The results of the present investigation revealed that several hydroquinones with redox potential in the microbial cells could also act as electron shuttle in the reduction mechanism and play a role in the formation of SNPs [17]. The mechanism of biosynthesis of SNPs is not completely understood, which has opened a door for exploring the better understanding of mechanism involved.

The FTIR spectra obtained shows the absorption spectral peaks located at $773,800,813,1040,1288,1530$, and $3226 / \mathrm{cm}$ in the region $500-4000 / \mathrm{cm}$ [Figure 4]. The analysis revealed the presence of $\mathrm{C}-\mathrm{OH}$ stretching vibrations, $\mathrm{C}-\mathrm{N}$ stretching vibrations of aliphatic and aromatic amines; $813 / \mathrm{cm}$ is assigned to S-O stretching of sulfonates [18], C-N stretching vibration at 1040.95/cm, 1288/cm assigned to the $\mathrm{O}-\mathrm{H}$ stretching vibration [19], $\mathrm{C}=\mathrm{N}$ peak at $1530 / \mathrm{cm}$. 


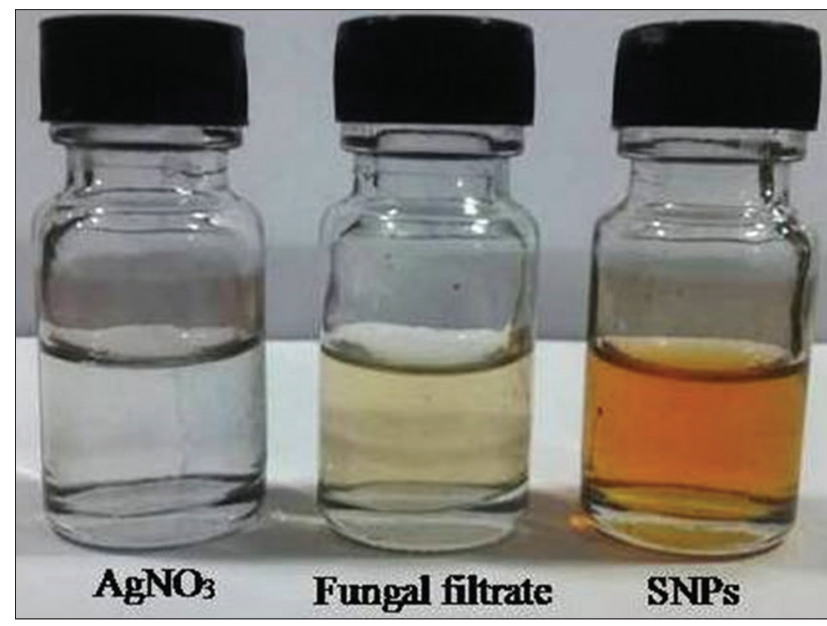

Figure 1: Color change in the synthesised silver nanoparticle.

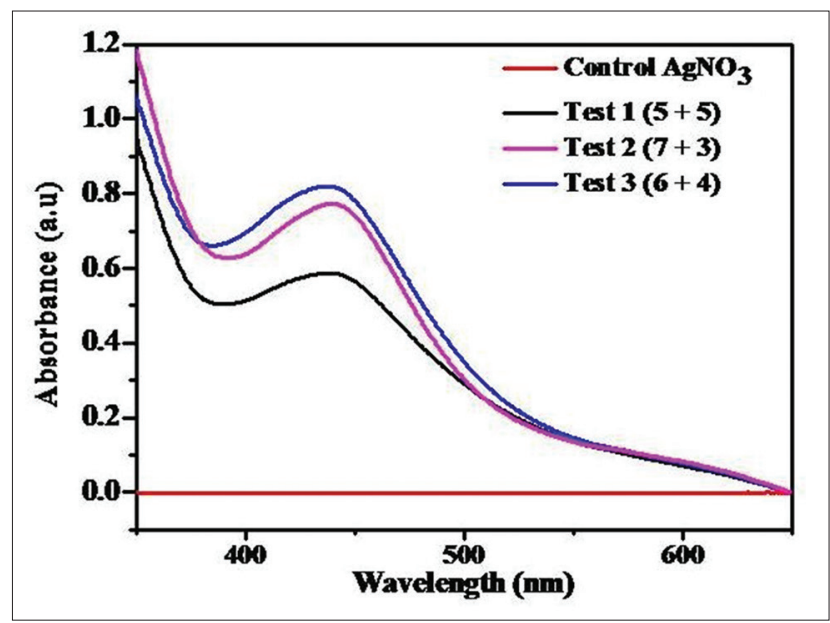

Figure 2: Ultraviolet-visible spectrum of silver nanoparticles (SNPs) showing the effect of different volumes on SNPs biosynthesis.

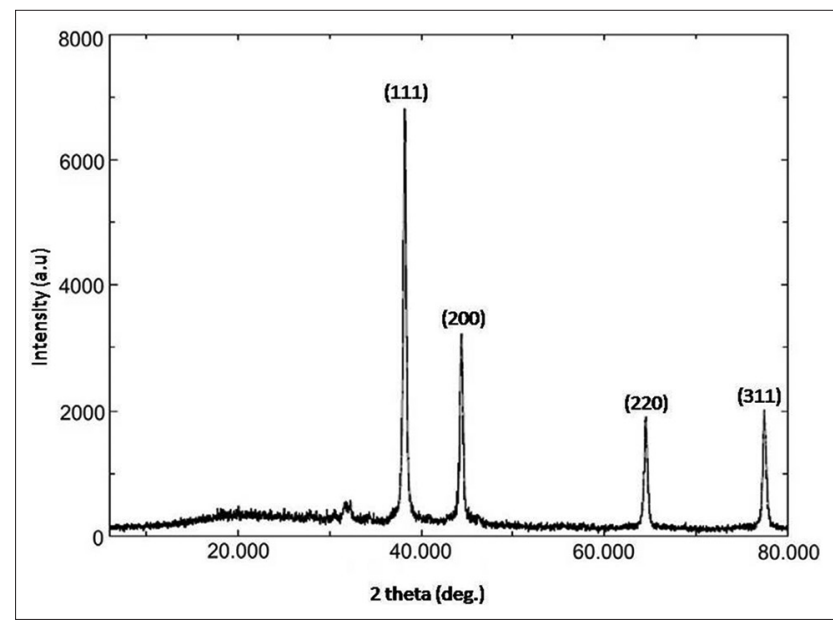

Figure 3: X-ray diffraction pattern of silver nanoparticles synthesized by Aspergillus awamori.

FTIR analysis reveals the presence of biomolecules and its different functional groups which reduce the silver nitrate and bind onto the NPs and helps in its stabilization.

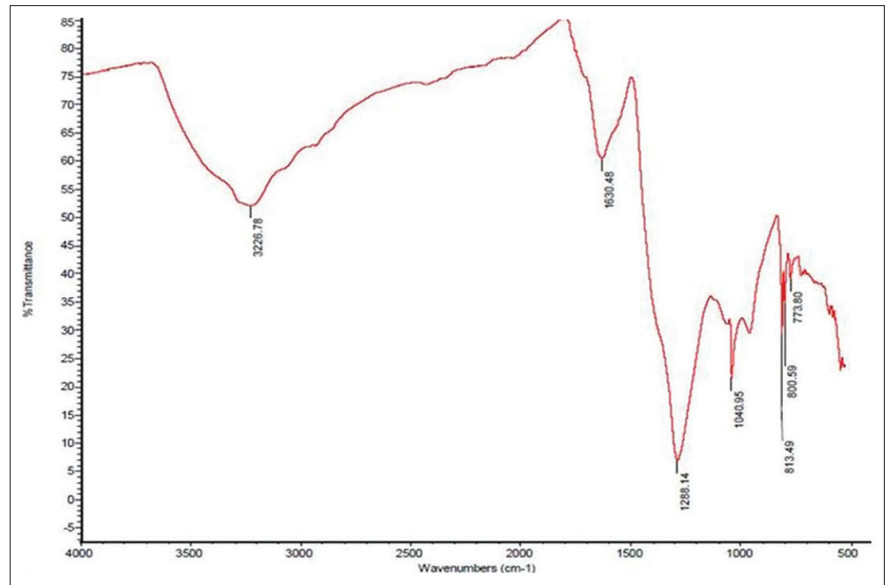

Figure 4: Fourier transform-infrared spectra of silver nanoparticles synthesized by Aspergillus awamori.

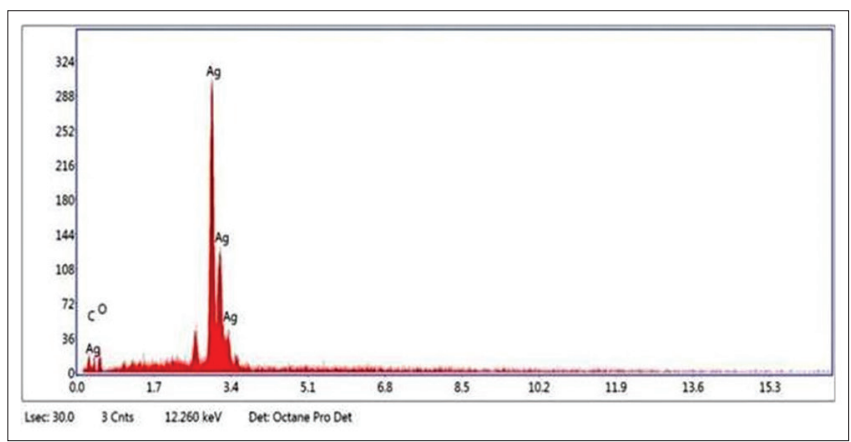

Figure 5: Energy dispersive X-ray analysis of silver nanoparticles.

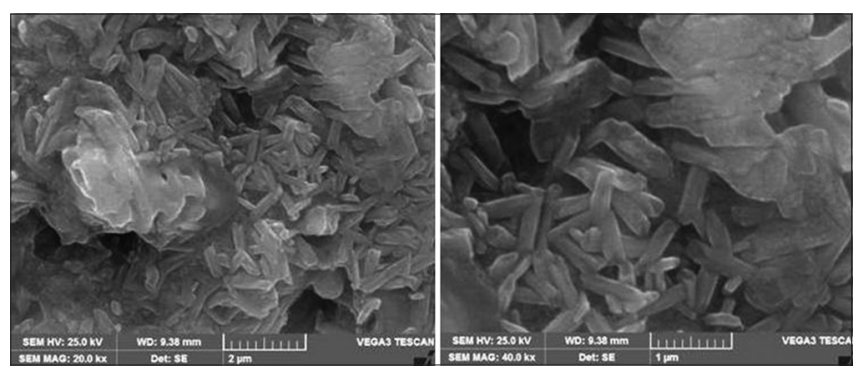

Figure 6: Scanning electron microscope image of silver nanoparticles formed at $1 \mathrm{mM}$ silver nitrate solution.

SNPs showed XRD pattern related to the crystalline structure with phase purity as shown in Figure 3. The distinct peaks positioned at $38.5^{\circ}, 44.4^{\circ}, 64.8^{\circ}$, and $77.6^{\circ}$ corresponding to $111,200,220$, and 311 , respectively, were observed as lattice planes for the face-centered cubic structure of SNPs (JCPDS 39-1346). The phase purity and high crystallinity are attributed to the strong and sharp diffraction peaks. The definite line broadening of the peaks suggests that the particles are in the nanometer range. Based on the Debye-Scherer's equation [20] the average crystallite size is calculated and was found to be $40 \mathrm{~nm}$.

The major constituents present in the as-synthesized NPs were analyzed by qualitatively and quantitatively using EDX analysis as shown in Figure 5. The peak is observed at $12.260 \mathrm{KeV}$, which is typical for the absorption of metallic SNPs [21]. Strong signals from the silver atoms are observed, while weaker signals from $\mathrm{C}$ atoms are also recorded. 

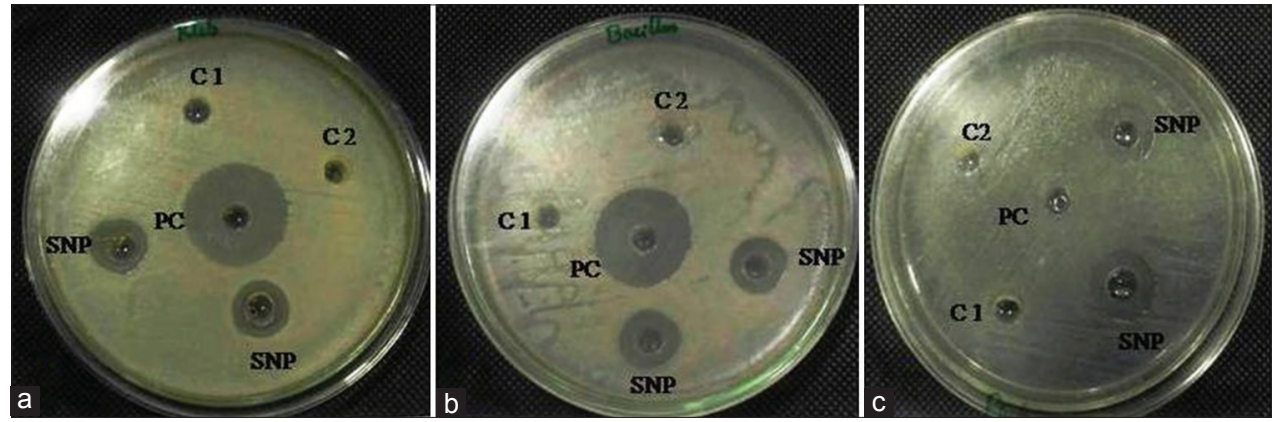

Figure 7: Antimicrobial activities of silver nanoparticles showing a zone of inhibition (a) Klebsiella pneumoniae, (b) Bacillus cereus,

(c) Pseudomonas aeruginosa.

The size and shape of SNPs NPs were examined by SEM analysis. As seen in Figure 6 the SNPs possess rod-like morphology with uniform size distribution within the range of $40-50 \mathrm{~nm}$.

The antibacterial activity of SNPs was tested by the well diffusion method against the pathogenic bacteria, P. aeruginosa, K. pneumoniae, and $B$. cereus. The antibacterial property of SNPs reveals the diameter of the zone of inhibition of $17 \mathrm{~mm}$ for P. aeruginosa, $15 \mathrm{~mm}$ for $K$. pneumoniae, and $17 \mathrm{~mm}$ for $B$. cereus. PC well-containing chloramphenicol shows a zone of $19 \mathrm{~mm}$ Figure 7. These results agreed with the previous work carried out by Khalil et al. and Li et al. $[3,5]$. The difference in the diameter of the zone of inhibition observed in these bacterial species is due to the difference in their cell wall composition [1]. The results were recorded as sensitive and intermediate as per the CLSI guidelines, 2013 for recommended for antibiotic depending on the zone of inhibition in $\mathrm{mm}$ diameter exhibited by each bacterial pathogen against antimicrobial agent $[1,22]$.

\section{CONCLUSION}

The present study reveals the need to explore new antibacterial agents in the era of emerging drug-resistant bacteria. The biosynthesized SNPs were found to be better antibacterial agents. Moreover, the concentration of SNPs also plays a crucial role in combating the pathogenic bacteria. The study highlights the antibacterial effect of NPs resulting in increased fold activity against drug-resistant pathogens conferring the emerging strategy to combat multidrugresistant microorganisms.

\section{ACKNOWLEDGMENTS}

The authors wish to acknowledge the B.M.S College of Engineering, Bengaluru, Karnataka, India, for SEM and FT-IR analysis of NPs. The authors also acknowledge the Department of Microbiology, Maharani's Science College for Women, Bengaluru, Karnataka, India, for providing laboratory facility.

\section{REFERENCES}

1. Keshavamurthy M, Srinath BS, Rai VR. Phytochemicals mediated green synthesis of gold nanoparticles using Pterocarpus santalinus L. (Red Sanders) bark extract and their antimicrobial properties. Part Sci Technol Int J 2017. DOI: org/10.1080/02726351 .2017 .1302533

2. Bhattacharya D, Gupt RK. Nanotechnology and potential of microorganisms. Crit Rev Biotechnol 2005;25:199-204.

3. Khalil MM, Ismail EM, El-Baghdady KZ, Mohamed D. Green synthesis of silver nanoparticles using olive leaf extract and its antibacterial activity. Arab J Chem 2013;7:1131-9.

4. Wijnhoven SW, Peijnenburg WJ, Herberts CA, Hagens WI, Oomen AG, Heugens EH, et al. Nano-silver: A review of available data and knowledge gaps in human and environmental risk assessment. Nanotoxicology 2009;3:109.

5. Li WR, Xie XB, Shi QS, Duan SS, Yang O, Chen YS. Antibacterial effect of silver nanoparticles on Staphylococcus aureus. Biometals 2011;24:135-41.

6. Baker S, Rakshith D, Kavitha KS, Santosh P, Kavitha HU, Rao Y, et al. Plants emerging as nanofactories towards facile route in synthesis of nanoparticles. Bio Impacts 2013;3:111-7.

7. Awwad AM, Salem NM, Abdeen AO. Green synthesis of silver nanoparticles using carob leaf extract and its antibacterial activity. Int J Ind Chem 2013;4:29.

8. Ahmad A, Mukherjee P, Senapati S, Mandal D, Khan MI, Kumar R, et al. Extracellular biosynthesis of silver nanoparticles using the fungus Fusarium oxysporum. Colloids Surf B Biointerfaces 2003;28:313-8.

9. Bhainsa KC, D'Souza SF. Extracellular biosynthesis of silver nanoparticles using the fungus Aspergillus fumigatus. Colloids Surf B Biointerfaces 2006;47:160-4.

10. Basavaraja S, Balaji SD, Lagashetty A, Rajasab AH, Venkataraman A. Extracellular biosynthesis of silver nanoparticles using the fungus Fusarium semitectum. Mat Res Bull 2008;43:1164-70.

11. Gade AK, Bonde P, Ingle AP, Marcato PD, Durán N, Rai MK. Exploitation of Aspergillus niger for synthesis of silver nanoparticles. J Biobased Mater Bioenerg 2008;2:243-7.

12. Balaji DS, Basavaraja S, Deshpande R, Mahesh DB, Prabhakar BK, Venkataraman A. Extracellular biosynthesis of functionalized silver nanoparticles by strains of Cladosporium cladosporioides fungus. Colloids Surf B Biointerfaces 2009;68:88-92.

13. Shaligram NS, Bule M, Bhambure R, Singhal RS, Singh SK, Szakacs G, et al. Biosynthesis of silver nanoparticles using aqueous extract from the compactin producing fungal strain. Proc Biochem 2009;44:939-43.

14. Verma VC, Kharwar RN, Gange AC. Biosynthesis of antimicrobial silver nanoparticles by the endophytic fungus Aspergillus clavatus. Nanomedicine (Lond) 2009;5:33-40.

15. Pilli R, Siddalingeshwara KG. Rapid confirmation and molecular identification of alkaline protease producing Aspergillus awamori through submerged fermentation. Int J Curr Microbiol Appl Sci 2016;5:1114-24.

16. Shankar SS, Rai A, Ahmad A, Sastry M. Rapid synthesis of Au, Ag, and bimetallic Au core-Ag shell nanoparticles using neem (Azadirachta indica) leaf broth. J Colloid Interface Sci 2004;275:496-502.

17. Saifuddin N, Wong CW, Yasumira AA. Rapid biosynthesis of silver nanoparticles using culture supernatant of bacteria with microwave irradiation. E-J Chem 2009;6:61-70.

18. Shanmugam R, Chelladurai M, Gnanajobitha G, Gurusamy A. 
Seaweed-mediated synthesis of gold nanoparticles using turbinaria conoide and its characterization. J Nanostruct Chem 2013;3:44.

19. Yildirim OA, Durucan C. Effect of precipitation temperature and organic additives on size and morphology of $\mathrm{ZnO}$ nanoparticles. J Mater Res 2012;27:1452-61.

20. Krishna RH, Nagabhushana BM, Nagabhushana H, Murthy NS, Sharma SC, Shivakumara C, et al. Effect of calcination temperature on structural, photoluminescence, and thermoluminescence properties of Y2O3: Eu3+ nanophosphor. J Phys Chem C 2013;117:1915.

21. Magudapathy P, Gangopadhyay P, Panigrahi BK, Nair KG, Dhara S. Electrical transport studies of $\mathrm{Ag}$ nano-clusters embedded in glass matrix. Phy B 2001;299:142-6.
22. Vishwanatha $T$, Keshavamurthy $M$, Siddalingeshwara $K G$, Kavyashree D. In vitro efficacy of lytic bacteriophage against antibiotic resistant bacterial pathogens. Int J Curr Microbiol Appl Sci 2016;5:642-8.
How to cite this article:
Vishwanatha T, Keshavamurthy M, Mallappa M, Murugendrappa M V,
Nadaf Y F, Siddalingeshwara K G and Dhulappa A. Biosynthesis,
characterization, and antibacterial activity of silver nanoparticles from
Aspergillus awamori. J. App Biol Biotech. 2018;6(05): 12-16.
DOI: $10.7324 / \mathrm{JABB} .2018 .60502$ 